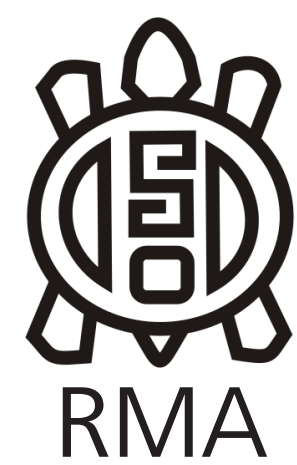

Arqueología

\title{
Análisis arqueobotánico de porotos carbonizados recuperados en el sitio tardío La Estancia (Catamarca, Argentina)
}

\author{
Archaeobotanical analysis of carbonized beans recovered in the late \\ site La Estancia (Catamarca, Argentina)
}

Juana Fuertes* y Karen Liotta**

\begin{abstract}
*Laboratorio de Análisis Cerámico, Facultad de Ciencias Naturales y Museo, Universidad Nacional de La Plata, Argentina. E-mail: juafuertes@hotmail.com **Laboratorio de Análisis Cerámico, Facultad de Ciencias Naturales y Museo, Universidad Nacional de La Plata, Argentina. E-mail: karenliotta.a@gmail.com
\end{abstract}

\begin{abstract}
Resumen
El objetivo del presente trabajo es analizar los restos vegetales carbonizados de Phaseolus spp. recuperados en el recinto 13 del sitio La Estancia, un poblado arqueológico del Valle de Hualfín, localizado en el municipio de La Puerta de San José (Prov. de Catamarca). La Estancia está constituido por 13 recintos cerrados, espacios semiabiertos y muros de contención construidos con pirca emplazados sobre una lomada. Las dataciones radiocarbónicas obtenidas para este sitio ubican su ocupación principalmente en el siglo XV, es decir, en los momentos finales del desarrollo cultural prehispánico regional. La muestra bajo análisis está conformada por 28 cotiledones y semillas que se identifican taxonómicamente sobre la base de sus caracteres externos macroscópicos: forma, textura externa, textura interna, confluencia de los márgenes, forma del contorno, forma de las caras y forma del ápice; y morfométricos: ancho y espesor. Mediante el empleo de material de referencia y la corroboración con bibliografía e ilustraciones de la temática se determinó que la muestra pertenece a Phaseolus vulgaris var. vulgaris $L$.
\end{abstract}

Palabras Clave: Valle de Hualfín; Período tardío; Análisis macroscópico; Phaseolus vulgaris; Identificación taxonómica.

\begin{abstract}
The aim of this work is to analyze the carbonized remains of Phaseolus spp. recovered in the structure number 13 of the site La Estancia, an archeological settlement of Hualfín valley located in La Puerta de San José (Prov. of Catamarca). La Estancia is constituted by 13 enclosures, semi-open spaces and retaining walls built with stone wall (pirca) placed on a knoll. The radiocarbon date obtained for this site places it occupation mainly in the XV century, in the final moments of the regional prehispanic cultural development. The sample under analysis is formed by 28 cotyledons and seeds that are taxonomically identified on the basis of their macroscopic external characters: form, extern texture, intern texture, confluence of the margins, form of the faces, form of the apex; and the morphometric characters; width and thickness. By the use of reference material and the corroboration with bibliography and illustrations of the theme it was determined that the sample belongs to the taxón Phaseolus vulgaris L. var. vulgaris.
\end{abstract}

Keywords: Hualfín Valley; Late period; Macroscopic analysis; Phaseolus vulgaris; Taxonomic identification.

El poroto común cultivado (Phaseoulus vulgaris var. vulgaris L.), dada su amplia distribución y adaptación, ha tenido un rol importante en la alimentación de las poblaciones de Argentina, Bolivia, Perú, Guatemala y México. Su domesticación se registra en Mesoamérica y en el área andina sudamericana a partir de una diversidad de especies americanas silvestres, y deriva en particular en el noroeste argentino de Phaseolus vulgaris var. aborigineus (Burk.) Baudet (Berglund-Brücher y Brücher 1976).
El presente trabajo tiene como propósito presentar los resultados obtenidos a partir del análisis de semillas y cotiledones carbonizados completos y fragmentados de Phaseolus spp. recuperados en el recinto 13 del sitio arqueológico La Estancia. Para tal finalidad se realiza la identificación taxonómica de la muestra sobre la base de sus caracteres morfométricos y morfológicos, siguiendo a Calo (2010), y la comparación con una muestra de referencia propia de porotos actuales. Los resultados se corroboran con ilustraciones y bibliografía de la temática 


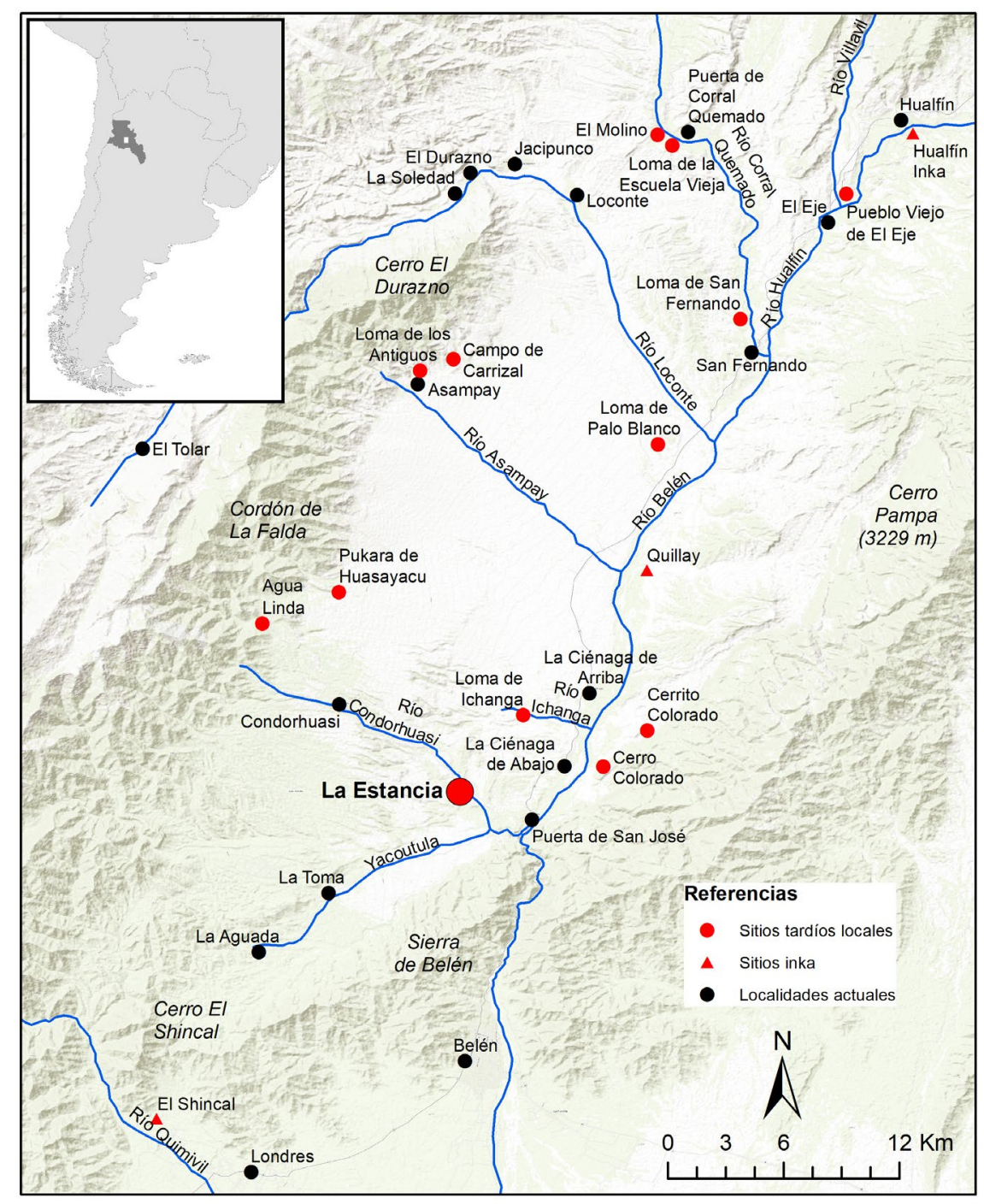

Figura 1. Mapa del Valle de Hualfín, con el sitio arqueológico La Estancia y los restantes sitios conocidos hasta el momento.

Figure 1. Hualfín Valley map, with the archaeological site La Estancia and the remaining sites known until now.

Los suelos aptos para el cultivo del poroto común, según el Sistema Nacional Argentino de Vigilancia y Monitoreo de plagas (2018), deben poseer una permeabilidad moderada, buen drenaje, texturas franco limoso grueso, franco arenoso o franco, gran contenido de material orgánico, estructura granular y un $\mathrm{pH}$ neutro. La temperatura de crecimiento es entre los 25 y $20^{\circ} \mathrm{C}$ entre el día y la noche, ya que los valores extremos afectan negativamente su actividad metabólica y su maduración. Este cultivo se adapta bien desde los 200 hasta los 1500 msnm y requiere un balance hídrico que contemple períodos alternados de humedad y secos para su desarrollo óptimo. Actualmente, su producción se localiza en el noroeste argentino, particularmente en las provincias

(Burkart 1952; Pochettino y Scattolin 1991; Babot et al 2007; Lema 2009; Medina et al. 2009; Calo 2010; Oliszweski y Babot 2015).

\section{Antecedentes y condiciones para el cultivo del poroto común}

El crecimiento de las variedades silvestres de Phaseolus vulgaris se restringe a la faja de selva mesotérmica de la ladera este de los Andes (Berglund-Brücher y Brücher 1976). Sus parientes domesticadas se cultivan en las zonas de valles y faldas templadas (Parodi 1991)

Numerosas investigaciones realizadas en el noroeste argentino y en las Sierras de Córdoba han evidenciado el manejo de la planta de Phaseolus spp., tanto en su variedad cultivada como silvestre, por parte de las poblaciones locales desde los momentos tempranos del Holoceno Tardío (Pochettino y Scattolin 1991; Carrizo et al. 1999; Korstanje y Würschmidt 1999; Albeck 2000; Babot 2004; Oliszewski 2004; López 2005; Medina y López 2005; Medina et al. 2009; Calo 2010; Miguez et al. 2010; Oliszewski y Babot 2015; Recalde y López 2017; López 2017). de Salta, Santiago del Estero, Jujuy, Tucumán y Catamarca. En particular, en la provincia de Catamarca, la producción de este cultivo se concentra en los departamentos de Santa Rosa y El Alto (Volante et al 2001).

\section{El sitio La Estancia en el Valle de Hualfín}

Los momentos tardíos prehispánicos del Noroeste argentino se caracterizaron por procesos sociales que condujeron a la intensificación agrícola (Lagiglia 2001; Oliszewski 2012). A partir del despliegue de una arquitectura especializada como canchones, terrazas de cultivo, andenería y construcciones agrohidráulicas, se evidencia un aumento demográfico y el incremento de la producción en términos regionales (Balesta et al. 2011; Capparelli et al. 2011; Williams et al. 2010, entre otros). En particular, en la región del Valle de Hualfín (Figura 1) en los sitios Campo de Carrizal, Azampay, Loma de los Antiguos, La Ciénaga, Cerro Colorado, Loma de Ichanga y La Estancia, se materializaron los procesos de producción y consumo de especies vegetales cultivadas y recolectadas como Zea mays L., Prosopis spp., Arachis hypogaea L., Phaseolus spp. (Balesta et al. 2011; Balesta et al. 2014; Balesta y Zagorodny 2018; Valencia et al. 2016 a). 
El Valle de Hualfín, como se lo conoce en la literatura arqueológica, corresponde geográficamente a la cuenca del río Belén, ubicada en el centro-oeste catamarqueño. Se extiende desde la localidad actual de Los Nacimientos en el Norte, hasta la de Puerta de San José hacia el Sur. El área desciende desde los 2.200 msnm al norte a los 1.400 en el sur, alcanzando las sierras más altas los $4.000 \mathrm{msnm}$ Desde el punto de vista fitogeográfico, corresponde a la Región Neotropical, dominio Chaqueño, provincia de Monte (Cabrera 1976; Morlans 1985), con predominio de matorral o estepa arbustiva xerófila, xamnófila o halófila. Abundan representantes de los géneros Prosopis spp. y Larrea spp., y de las especies arbóreas Acacia furcatispina, Senegalia gilliesii y Cericidium praecox. La región es semiárida y de clima cálido, continental (Morello 1958), con precipitaciones entre los 200 y $400 \mathrm{~mm}$ por año de carácter estival concentradas por debajo de los 1500 msnm de altitud (Ardissone 1961), y con una temperatura media anual de $20^{\circ} \mathrm{C}$ (Valencia y Balesta 2013).

La Estancia (Figura 2) es un poblado arqueológico del sector sur del Valle de Hualfín, con una ocupación ubicada cronológicamente -al menos en sus momentos finales- en el siglo XV (Wynveldt et al. 2017). Está conformado por 13 estructuras emplazadas sobre una lomada entre las que se destacan recintos aislados, espacios semiabiertos y muros de contención, construidos con piedra pircada (Zagorodny et al. 2015). El recinto 13 es una estructura rectangular de unos $21 \mathrm{~m}^{2}$ sin abertura de acceso. Para su excavación se dividió la estructura en 9 cuadrículas de 1,40 x 1,70 m cada una, que fueron excavadas por niveles artificiales de $10 \mathrm{~cm}$. La recuperación de los materiales se realizó mediante técnica manual y con zarandas con una luz de 1 y $5 \mathrm{~mm}$. Entre los materiales recuperados se identificaron restos óseos, fragmentos de cerámica y una gran cantidad de restos vegetales carbonizados -ramas, marlos, mazorcas y granos de maíz (Zea mays) (Fuertes 2019; Valencia et al. 2016 b) y semillas de otras especies vegetales, como poroto (Phaseolus spp.)-. El peso total de estos macrorrestos vegetales es de 2368 gramos, que se corresponde a 39 litros. Estos valores no tienen antecedentes hasta el momento en ninguno de los otros sitios tardíos del valle. En este trabajo se analizan semillas y cotiledones carbonizados completos y fragmentados de Phaseolus spp. recuperados en el recinto 13, con el propósito de contribuir al conocimiento sobre las prácticas de producción agrícola del paisaje tardío del Valle del Hualfín.

\section{Materiales y Métodos}

Del total de los macrorrestos vegetales hallados en el recinto 13, se tomó al azar una muestra constituida por material proveniente de los niveles 50 a $80 \mathrm{~cm}$ de las cuadrículas C2 y C3. La muestra analizada constó de 28 ejemplares carpológicos carbonizados. Su identificación taxonómica se realizó sobre la base de la metodología propuesta por Calo (2010), donde se tuvieron en cuenta los caracteres externos macroscópicos y los caracteres morfométricos. La muestra fue observada a ojo desnudo y con lupa binocular hasta 40x (aumentos).

En relación con los caracteres externos se consideró: 1) el órgano representado: parte de la planta analizada para su determinación taxonómica que puede tomar el valor de pericarpo (endocarpo, mesocarpo, epicarpo), semilla y/o cotiledón; 2) el estado de conservación que refiere al porcentaje conservado de cada ejemplar, completo (100\%), semicompleto (>50\%) o fragmentario ( $\leq 50 \%)$ y 3) los caracteres morfológicos, entre los que se analizó:

a. Forma: forma tridimensional general del espécimen.

b. Textura de la superficie externa: caracteriza la cubierta seminal del ejemplar.

c. Textura de la superficie interna: refiere a las características de la superficie interior del cotiledón en los casos en que esta es visible

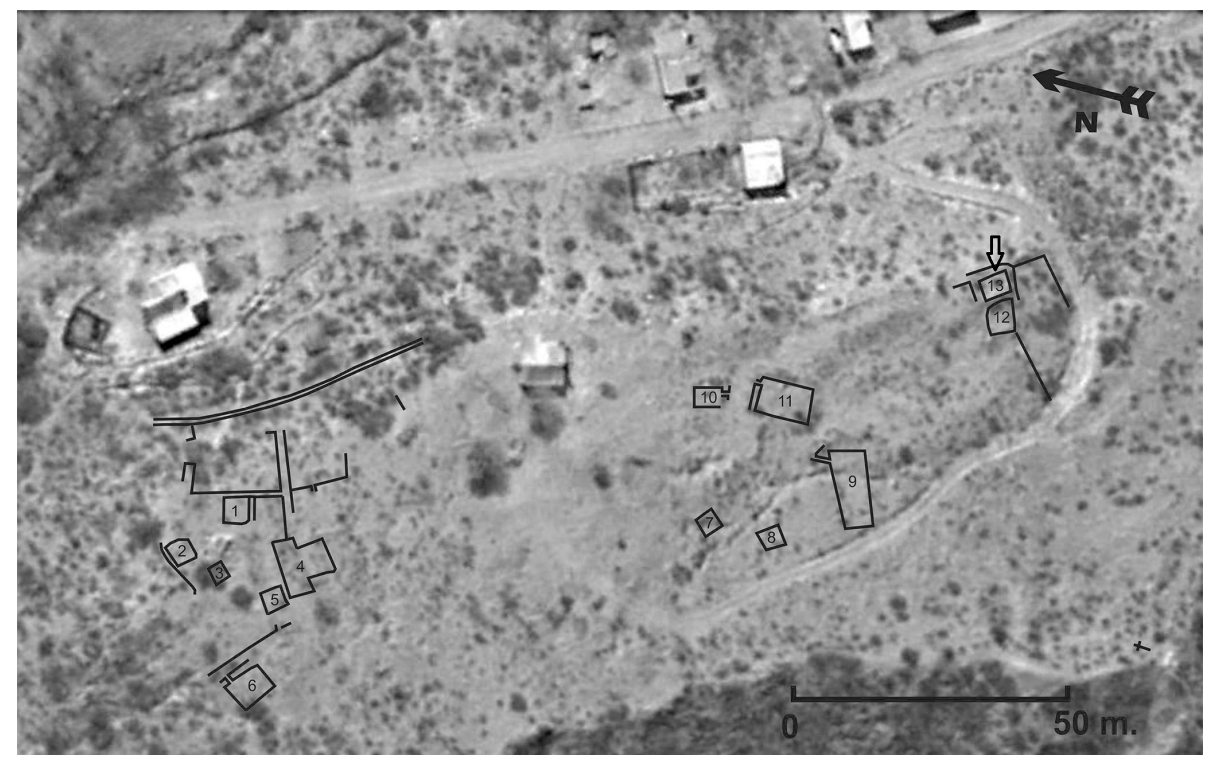

Figura 2. Plano del sitio arqueológico La Estancia realizado sobre su imagen satelital con el recinto 13 señalado.

Figure 2. Map of the archaeological site La Estancia realized on its satellite image with the structure number 13 indicated. 
d. Configuración de los márgenes: forma adquirida en la zona de convergencia de las caras del ejemplar que puede derivar en formas redondeadas, truncadas, convexas o cóncavas.

e. Forma del contorno: diseño del borde del espécimen visto en dos dimensiones.

f. Forma de las caras: forma de los cotiledones vistos en corte transversal.

g. Forma de los ápices: morfología general de los extremos de la semilla.

h. Visibilidad de la radícula: muy visible, visible, poco visible, no visible.

i. Forma del embrión: indica el modo en que se dispone el embrión en la semilla.

j. Posición del hilo: lugar de la semilla donde se encuentra la marca de la separación con el funículo.

k. Presencia o ausencia de la línea fisural.

Como caracteres morfométricos se analizaron solamente ancho y espesor debido a que la fragmentación de un gran porcentaje de ejemplares arqueológicos imposibilita la medición del largo. Estos valores se midieron con calibre electrónico y se expresaron en milímetros.

Con la finalidad de realizar una determinación infraespecífica de la muestra y distinguir entre porotos silvestres (Phaseolus vulgaris var. aborigineus (Burk.) Baudet) y cultivados (Phaseolus vulgaris var. vulgaris), los datos fueron comparados con la clasificación propuesta por Babot y colaboradores (2007), que toma como caracteres diagnósticos la presencia de micrópila y las dimensiones del espécimen. En líneas generales, las variedades cultivadas presentan dimensiones grandes (9,95-13,23 $\mathrm{mm}$ de largo, 6,9 $\mathrm{mm}$ de ancho y 5,49-5,5 $\mathrm{mm}$ de espesor), y presentan micrópila. En cambio, los silvestres presentan dimensiones más pequeñas, 6,68$8,54 \mathrm{~mm}$ de largo, 4,72-5,9 $\mathrm{mm}$ de ancho y 2,18-3,14 $\mathrm{mm}$ de espesor.

El análisis estadístico descriptivo se realizó considerando las variables ancho y espesor de ambas muestras. Para la medición de la variable espesor se tuvieron en cuenta los cotiledones. Se determinó para cada una la media, la mediana, el desvío estándar y la varianza (Kelmansky, 2009). Con el fin de examinar si las muestras presentan diferencias significativas en el ancho y en el espesor, se utilizó la prueba t de student al 0,5 para dos muestras suponiendo varianzas iguales. La implementación de esta prueba resulta pertinente ya que permite estimar las diferencias presentes entre partes de dos poblaciones distintas, específicamente para muestras de pequeño tamaño que presentan una distribución normal, a partir de la prueba de hipótesis de medias usando la distribución T (Sokal y Rohlf 1986).

Los resultados fueron corroborados con bibliografía e ilustraciones de la temática, con foco en los desarrollos argentinos que toman como referencia especies locales (Burkart 1952; Pochettino y Scattolin 1991; Babot et al 2007; Lema 2009; Medina et al. 2009; Calo 2010; Oliszweski y Babot 2015). Los materiales arqueológicos se compararon con una muestra de referencia propia, constituida por 60 ejemplares actuales provenientes de la provincia de Salta que representan tres cultivares distintos. Se expuso la mitad a un proceso de carbonización para la posterior comparación de muestras en un mismo estado. La carbonización se realizó en fogón a llama directa con un rango de temperatura entre $500^{\circ} \mathrm{C}$ y $700^{\circ} \mathrm{C}$ y en atmósfera anaeróbica, durante cuatro minutos, tiempo que llevó su completa carbonización.

\section{Resultados}

El análisis de los caracteres macroscópicos externos de la muestra de referencia termoalterada evidencia que los tres cultivares de porotos actuales comparten los atributos que se mencionan a continuación: formas generales ovada, ovalada y arriñonada; textura externa rugosa, escamosa, lisa o plegada; textura interna lisa o estriada; configuración de los márgenes cóncava; contorno liso y redondeado; caras planas o cóncavas; ápice rendondeado; radícula visible o poco visible; embrión doblado; hilo en una posición lateral; y ausencia de línea fisural. Los valores obtenidos mediante la medición de los
Figura 3. Esquema de los caracteres morfológicos utilizados para la identificación taxonómica de las semillas y cotiledones de Phaseolus spp. arqueológicos, siguiendo a Calo (2010).

Figure 3. Scheme of the morphological characters used to the taxonomic identification of the seeds and cotyledons of archaeological Phaseolus spp., following Calo (2010).
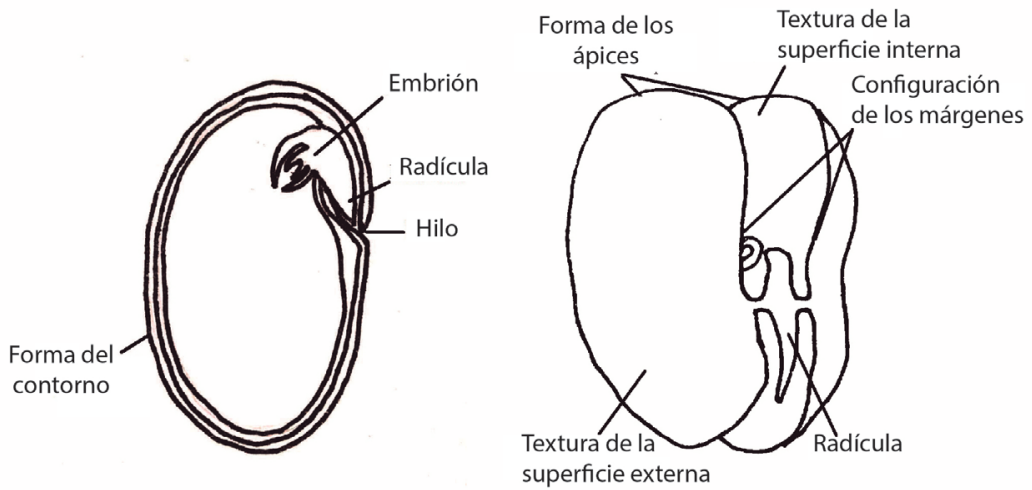
Figura 4. Ejemplares de la muestra arqueológica de Phaseolus vulgaris var. vulgaris analizada.

Figure 4. Specimens of the archaeological sample analyzed of Phaseolus vulgaris var. vulgaris
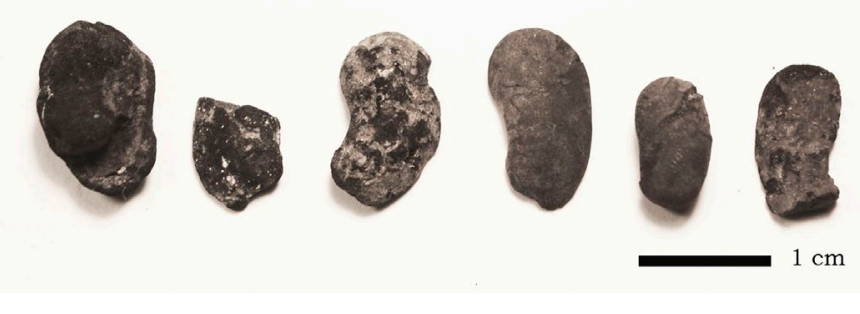

caracteres morfométricos se sintetizaron en la Tabla 1.

Los caracteres macroscópicos externos de la muestra arqueológica presentan como atributos en común:

1. Forma: ovalada, cuadrangular, arriñonada, fusiforme. Se identificaron restos que presentan rasgos combinados: ovalado-arriñonado y ovalado-cuadrangular.

2. Textura externa: lisa, irregular, con agrietamiento, con oquedades, verrugosa, fragmentada, erosionada.

3. Textura interna: lisa, verrugosa, globular, irregular, estriada, erosionada.

4. Confluencia de los márgenes: convexo, cóncavo.

5. Forma del contorno: redondeado, irregular.

6. Forma de las caras: cóncavo, redondeado, recto.

7. Forma del ápice: redondeado.

Los órganos representados son semillas y cotiledones con estado de conservación completo y semicompleto. Debido al proceso de termoalteración y al grado de fragmentación, sólo en algunos ejemplares se observa un embrión doblado, la radícula o su negativo, el hilo en posición lateral, y carencia de la línea fisural. Estos atributos son propios del género Phaseolus spp. (Burkart 1952; Pochettino y Scattolin 1991; Babot et al 2007; Calo 2010)

La comparación con el material de referencia permite determinar que el $75 \%$ de la muestra pertenece a Phaseolus vulgaris var. vulgaris (Figura 3), mientras que el porcentaje restante se identifica a nivel género, debido a que en algunos casos, la termoalteración dificulta visualizar los rasgos morfológicos que permiten la identificación infragénero.
La estadística descriptiva (Tabla 1) muestra valores similares respecto a la media, la mediana y la varianza del ancho y del espesor en ambas muestras. Los valores de media y la mediana no presentan diferencias sustanciales y expresan una distribución homogénea. La varianza y el desvío estándar poseen una dispersión similar y pequeña con respecto a la media. Las pruebas de t student (Tabla 2) no evidenciaron diferencias significativas entre los dos tipos de muestras para el ancho y espesor.

\section{Discusión y Conclusiones}

Gran parte de los restos carbonizados de Phaseolus spp. recuperados en el recinto 13 del sitio La Estancia se identificaron como pertenecientes a la variedad de poroto cultivado (Phaseolus vulgaris var. vulgaris). Los atributos observados y analizados a partir de la identificación taxonómica evidencian, en términos generales, ciertos rasgos morfológicos y dimensionales en común en relación a la forma, textura y tamaño.

En lo que respecta a las variables cuantitativas, las dimensiones de los especímenes analizados coinciden con los rangos de tamaño $(6,14-7,7 \mathrm{~mm}$ de ancho y 2,733,53 mm de espesor) determinados a partir de la muestra de referencia constituida por porotos carbonizados, así como también con sus formas generales. El análisis estadístico realizado muestra que no existen diferencias significativas en el ancho y en el espesor entre la muestra arqueológica y la muestra de referencia de Phaseolus vulgaris var. vulgaris. Los resultados derivados de estos estudios respaldan la idea de que los restos de Phaseolus vulgaris recuperados en el sitio La Estancia son cultivados. Asimismo, retomando los rangos propuestos por Babot y colaboradores (2007) para identificar variedades cultivadas en estado seco $(6,90 \mathrm{~mm}$ de ancho y 2,18-5,55

\begin{tabular}{|c|c|c|c|c|c|c|c|c|}
\hline \multicolumn{10}{|c|}{ Ancho } \\
\hline Muestra & N & Mínimo & Máximo & Media & Mediana & Varianza & $\begin{array}{c}\text { Desvío } \\
\text { Estandar }\end{array}$ \\
\hline Arqueológica & 28 & 4,94 & 8,23 & 6,78 & 6,86 & 0,97 & $(+/-) 0,98$ \\
\hline $\begin{array}{c}\text { De referencia } \\
\text { Carbonizada }\end{array}$ & 30 & 4,92 & 8,14 & 6,92 & 7 & 0,61 & $(+/-) 0,78$ \\
\hline \multicolumn{8}{|c|}{ Espesor } \\
\hline Muestra & $\mathrm{N}$ & Mínimo & Máximo & Media & Mediana & Varianza & $\begin{array}{c}\text { Desvío } \\
\text { Estandar }\end{array}$ \\
\hline Arqueológica & 28 & 1,96 & 6,57 & 2,9 & 2,94 & 0,16 & $(+/-) 0,4$ \\
\hline $\begin{array}{c}\text { De referencia } \\
\text { Carbonizada }\end{array}$ & 30 & 2,92 & 7,23 & 3,13 & 3,13 & 0,16 & $(+/-) 0,4$ \\
\hline
\end{tabular}

Tabla 1. Estadísticas descriptivas para ancho y espesor de semillas carbonizadas de Phaseolus vulgaris arqueológicas y actuales.

Table 1. Descriptive statistics for width and thickness of archaeological and present carbonized Phaseolus vulgaris seeds. 


\begin{tabular}{|l|c|c|c|c|}
\hline \multicolumn{5}{|c|}{ Prueba t para dos muestras suponiendo varianzas iguales } \\
\hline & $\begin{array}{c}\text { Muestra } \\
\text { Arqueológica - } \\
\text { Ancho }\end{array}$ & $\begin{array}{c}\text { Muestra de } \\
\text { Referencia } \\
\text { Carbonizada- } \\
\text { Ancho }\end{array}$ & $\begin{array}{c}\text { Muestra } \\
\text { Arqueológica - } \\
\text { Espesor }\end{array}$ & $\begin{array}{c}\text { Muestra de } \\
\text { Referencia } \\
\text { Carbonizada- } \\
\text { Espesor }\end{array}$ \\
\hline Media & 2,907857143 & 2,973333333 & 2,907857143 & 2,973333333 \\
\hline Varianza & 0,165787831 & 0,163216092 & 0,165787831 & 0,163216092 \\
\hline $\mathrm{N}$ & 28 & 30 & 28 & 30 \\
\hline $\begin{array}{l}\text { Varianza } \\
\text { agrupada }\end{array}$ & 0,164456037 & & 0,164456037 & \\
\hline $\begin{array}{l}\text { Diferencia } \\
\text { hipotética de } \\
\text { las medias }\end{array}$ & 0 & & 56 & \\
\hline $\begin{array}{l}\text { Grados de } \\
\text { libertad }\end{array}$ & 56 & & $-0,614446591$ & \\
\hline Estadístico $t$ & $-0,614446591$ & & 0,270704382 & \\
\hline $\begin{array}{l}\mathrm{P}(\mathrm{T}<=\mathrm{t}) \text { una } \\
\text { cola }\end{array}$ & 0,270704382 & & 1,672522303 & \\
\hline $\begin{array}{l}\text { Valor crítico de } \\
\mathrm{t} \text { (una cola) }\end{array}$ & 1,672522303 & & 0,541408763 & \\
\hline $\begin{array}{l}\mathrm{P}(\mathrm{T}<=\mathrm{t}) \text { dos } \\
\text { colas }\end{array}$ & 0,541408763 & & 2,003240719 & \\
\hline $\begin{array}{l}\text { Valor crítico de } \\
\mathrm{t} \text { (dos colas) }\end{array}$ & 2,003240719 & & & \\
\hline
\end{tabular}

Tabla 2. Prueba $T$ de Student para ancho y espesor de semillas de Phaseolus vulgaris arqueológicas y actuales.

Table 2. Student's T test for width and thickness of archaeological and present Phaseolus vulgaris seeds. $\mathrm{mm}$ de espesor) podemos determinar similitudes con los observados en la muestra arqueológica analizada, a pesar de los cambios producidos por la termoalteración.

Se registraron algunas tendencias en cuanto a la morfología, principalmente la forma arriñonada, ovalada y cuadrangular, que dan cuenta de posibles diferencias al interior de Phaseolus vulgaris var. vulgaris. En este sentido, resulta pertinente la posibilidad de profundizar en la determinación de estas diferencias a partir de la combinación de estrategias de análisis, teniendo en cuenta los aportes de los estudios microscópicos sobre Phaseolus vulgaris arqueológicos (Oliszewski y Babot 2015). Vemos necesaria la construcción de herramientas metodológicas para la identificación taxonómica de restos arqueobotánicos carbonizados que permitan profundizar en la interpretación de la producción agrícola en el área del noroeste argentino.

Durante el desarrollo del presente trabajo, surgieron diversos interrogantes. Con respecto a las tendencias observadas en la morfología de los especímenes analizados, éstas podrían corresponder a distintos cultivares de Phaseoulus vulgaris var. vulgaris, lo que conduce a pensar que existió un amplio conocimiento en torno al manejo de estos especímenes. Por otro lado, las condiciones edáficas, altitudinales y climáticas requeridas para el desarrollo óptimo de sus cultivos, sumado a que en la actualidad se registra su producción en Catamarca, permite pensar que pudieron existir condiciones aptas para que estos cultígenos fueran producidos en el Valle de Hualfín, debido a que su altura y clima lo permiten. La presencia de construcciones agrícolas y obras hidráulicas arqueológicas en el valle respaldaría esta idea. En este sentido, es interesante pensar el análisis de los restos arqueobotánicos en relación a los sitios donde se registran estructuras asociadas a la producción agrícola, con el objetivo de generar abordajes de carácter regional.
A partir de ello, cabe preguntarnos en qué lugares se desarrolló la producción, así como a qué escala y para quiénes. La esfera de lo productivo, también nos remite al consumo de alimentos; las prácticas asociadas al consumo nos llevan a preguntarnos acerca de las formas, los modos, los usos y los espacios en los cuales se desarrollaron. Estas inquietudes guiarán las futuras investigaciones tendientes al estudio de las prácticas agrícolas en el Valle de Hualfín.

La Plata, 10 de mayo de 2019

\section{Agradecimientos}

A la Lic. Celeste Valencia por su dedicación y acompañamiento en el desarrollo de este trabajo. A las Dras. María Emilia Iucci y Patricia Arenas por sus comentarios constructivos. A la Lic. Nora Inés Zagorodny por su mirada integradora y por brindarnos el espacio de trabajo. A los evaluadores por sus acertados comentarios.

\section{Bibliografía}

Albeck, M. E. 2000. La vida agraria en los Andes del Sur, en Nueva Historia Argentina, M. N. Tarragó, ed., pp. 187-228. Buenos Aires: Editorial Sudamericana.

Babot, M. del P. 2004. Tecnología y utilización de artefactos de molienda en el Noroeste prehispánico (Tesis doctoral, Universidad Nacional de Tucumán).

Babot, M. D. P., Oliszewski, N., y Grau, A. 2007. Análisis de caracteres macroscópicos y microscópicos de Phaseolus Vulgaris (Fabaceae, Faboideae) silvestres y cultivados del noroeste argentino: una aplicación en arqueobotánica. Darwiniana, nueva serie, 45(2), 149-162.

Balesta, B., Zagorodny, N. y Wynveldt, F. 2011. La configuración del paisaje Belén (valle de Hualfín, 
Catamarca). Relaciones de la Sociedad Argentina de Antropología, 36, 149-175.

Balesta, B., Valencia, C., y Wynveldt, F. 2014. Procesamiento de maíz en el tardío del Valle de Hualfín ¿Un contexto doméstico de producción de chicha? Arqueología, 20, 61-82.

Balesta, B. y Zagorodny, N. I. 2018. El tiempo práctico en el paisaje agrario del Valle de Hualfín, en Las dimensiones del paisaje tardío en el Valle de Hualfín, Federico Wynveldt y Bárbara Balesta, comps., pp. 75-102. Buenos Aires: Sociedad Argentina de Antropología.

Berglund-Brücher, O., y Brücher, H. 1976. The South American wild bean (Phaseolus aborigineus Burk.) as ancestor of the common bean. Economic Botany, 30(3), 257-272.

Burkart, A. 1952. Las leguminosas argentinas silvestres y cultivadas, $2^{a}$. Edición. Buenos Aires: Acme.

Calo, C. M. 2010. Plantas útiles y prácticas cotidianas entre los aldeanos al sur de los Valles Calchaquíes $(600$ aC-900 dC) (Tesis doctoral, Facultad de Ciencias Naturales y Museo).

Capparelli, A., Hilgert, N., Ladio, A., Lema, V. S., Llano, C., Molares, S., Pochettino, M.L. y Stampella, P. 2011. Paisajes culturales de Argentina: pasado y presente desde las perspectivas etnobotánica y paleoetnobotánica. Revista de la Asociación Argentina Ecología de Paisajes, 2(2), 67-79.

Carrizo, J., Cano, S. F. y Soler Nixdorff, M. M. 1999. Recursos vegetales comestibles en el Valle de Tafí durante el Período Formativo: análisis arqueobotánico del sitio Casas Viejas-El Mollar (STucTav2). En: Actas del XII Congreso Nacional de Arqueología Argentina (pp. 22-24). La Plata.

Figueroa, G. G. (2008). Los sistemas agrícolas del Valle de Ambato, Catamarca, siglos $\mathrm{VI}$ a XI dC. Intersecciones en Antropología, (9), 313-317.

Fuertes, J. 2019. Análisis de macrorrestos vegetales carbonizados de La Estancia, un sitio tardío del Valle de Hualfín (depto. de Belén, prov. de Catamarca). En prensa.

Kelmansky, D.M., 2009. Estadística para todos. Buenos Aires: Ministerio de Educación-Instituto Nacional de Educación Tecnológica.

Korstanje, M. A. y Würschmidt, A. 1999. Producir y recolectar en los valles altos del NOA: Ios Viscos como caso de estudio, en En los tres reinos: prácticas de recolección en el Cono Sur de América, C. Aschero, M. Korstanje y P. Vuoto, eds., pp. 151-160, San Miguel de
Tucumán: Magna Ediciones.

Lagiglia, H. 2001. Los orígenes de la agricultura en la Argentina, en Historia argentina prehispánica, E. E. Berberián y A. E. Nielsen, eds., Tomo 1, pp 41-81, Córdoba: Editorial Brujas.

Lema, V. 2009. Domesticación vegetal y grados de dependencia ser humano-planta en el desarrollo cultural prehispánico del noroeste argentino (Tesis doctoral, Facultad de Ciencias Naturales y Museo).

López, M. L. 2005 Los pobladores productores de alimentos de las sierras de Córdoba. Primeras evidencias arqueobotánicas de los sitios Tala Cañada 1 y C. Pun.39. La Zaranda de Ideas, 1, 89-91.

López, M. L. 2018. Archaeobotany in central Argentina: macro-and microscopic remains at several archaeological sites from early Late Holocene to early colonial times (3,000-250 bp). Vegetation history and archaeobotany, 27(1), 219-228.

Medina, M. E., López, M. L., y Berberián, E. E. 2009. Agricultura y recolección en el Tardío Prehispánico de las Sierras de Córdoba (Argentina): el registro arqueobotánico de C. Pun. 39. Arqueología, 15, 217-230.

Medina, M. y López, M. L. 2005 Evidencias prehispánicas de Phaseolus spp. en Puesto La Esquina 1 (Córdoba, Argentina). Arqueología 13, 241-245

Miguez, G., Arreguez, G. A., y Oliszewski, N. 2012. Primeros hallazgos de la forma doméstica del poroto común en el Piedemonte Tucumano ( $1^{\circ}$ milenio d. C.). Comechingonia, 16(1), 307-314.

Oliszewski, N. 2004. Utilización de recursos vegetales en Campo del Pucará (Andalgalá, Catamarca) durante el Período Formativo (200-500 d.C.). Análisis de macrorrestos (Tesis doctoral, Universidad Nacional de Tucumán).

Oliszewski, N. (2012). La variabilidad racial del maíz y los cambios sociales durante el 1 y 2 milenio dC en el Noroeste argentino, en Las Manos en la masa. Arqueologías, antropologías e historias de la alimentación en Suramérica, M. D. P. Babot, M. Marschoff y F. Pazzarelli, Eds., pp. 271-297, Córdoba: Universidad nacional de Córdoba. Facultad de Filosofía y Humanidades, Museo de Antropología UNC - Instituto Superior de Estudios Sociales UNT.

Oliszewski, N. y Babot, M. D. P. 2015. Procesos de selección del poroto común en los valles altos del Noroeste Argentino en tiempos prehispánicos. Análisis micro y macroscópico de especímenes arqueobotánicos, en Avances y desafíos metodológicos en arqueobotánica. 
Miradas consensuadas y diálogos compartidos desde Sudamérica, Carolina Belmar y Verónica Lema, eds., pp. 301-324. Chile: Monografías Arqueológicas Facultad De Patrimonio Cultural y Educación Universidad SEK.

Parodi, L. 1991. Agricultura prehispánica. San Salvador de Jujuy: Editorial de la Universidad Nacional de Jujuy.

Phaseolus vulgaris (05 de abril, 2019). Recuperado de: https://www.sinavimo.gov.ar/cultivo/phaseolus-vulgaris

Pochettino, M. L. y Scattolin, M. C. 1991. Identificación y significado de frutos y semillas carbonizados de sitios arqueológicos de la ladera occidental del Aconquija, Prov. Catamarca, Rca. Argentina. Revista Museo de La Plata, Antropología. 9: 169-181

Recalde, A. y López, L. 2017. Las sociedades prehispánicas tardías en la región septentrional del centro de Argentina (Sierras del Norte, Córdoba). Avances a su conocimiento desde los recursos vegetales. Chungará (Arica), 49(4), 573-588.

Sokal, R.R. and Rohlf, F.J., 1986. Introducción a la Bioestadística (Vol. 5). Reverté.

Valencia, M. C., Flores, M., Wynveldt, F. y Balesta, B. 2016 a. Identificación de variedades de maíz y prácticas agrícolas en el valle de Hualfín (Catamarca, Argentina). Revista Española de Antropología Americana, 46, 283304.
Valencia, M. C., Zagorodny, N., Balesta, B. y Liotta, K. 2016 b. El sitio La Estancia en la red del paisaje Tardío del Valle de Hualfín, en Actas del XIX Congreso Nacional de Arqueología Argentina, pp. 444-445. San Miguel de Tucumán: Facultad de Ciencias Naturales e I.M.L. Universidad Nacional de Tucumán.

Volante, J. N., Bianchi, A. R., Paoli, H. P., \& Fernandez, D. 2001. Monitoreo de Cultivos Extensivos del Noroeste Argentino a Partir de Sensores Remotos. Campaña de Trigo y Cártamo.

Williams, V., Korstanje, A., Cuenya, P. y Villegas, M. P. 2010. La dimensión social de la producción agrícola en un sector del Valle Calchaquí Medio, en Arqueología de la agricultura: Casos de estudio en la región andina argentina, A. Korstanje y M. Quesada, eds., pp. 178-207. San Miguel de Tucumán: Ediciones Magna.

Wynveldt, F., Balesta, B., Iucci, M., Valencia, C., y Lorenzo, G. 2017. Late Chronology in Hualfín Valley (Catamarca, Argentina): A Revision from 14C Dating. Radiocarbon 59(1): 91-107.

Zagorodny, N.; Balesta, B. y Wynveldt, F. 2015. Resultados preliminares de las investigaciones arqueológicas en el sitio La Estancia (Departamento Belén, Catamarca) en el marco de una experiencia educativa. Revista del Museo de La Plata. Sección Antropología, 14(88), 1-10. 